\title{
EPIDEMIOLOGIA DA HANSENÍASE E A DESCENTRALIZAÇÃO DAS AÇÕES DE CONTROLE NO BRASIL
}

\author{
LEPROSY EPIDEMIOLOGY AND THE \\ DECENTRALIZATION OF CONTROL ACTIONS IN BRAZIL
}

\section{LA EPIDEMIOLOGÍA DE LA LEPRA Y LA DESCENTRALIZACIÓN DE LAS ACCIONES DE CONTROL EN BRASIL}

\author{
Rayssa Nogueira Rodrigues ${ }^{1}$ \\ Ricardo Alexandre Arcêncio ${ }^{2}$ \\ Francisco Carlos Felix Lana ${ }^{3}$
}

Como citar este artigo: Rodrigues RN, Arcêncio RA, Lana FCF. Epidemiologia da hanseníase e a descentralização das ações de controle no Brasil. Rev baiana enferm. 2021;35:e39000.

Objetivo: analisar a situação epidemiológica da hanseníase no Brasil e sua associação com a descentralização das ações de controle. Método: estudo ecológico misto com dados secundários do Sistema de Informação de Agravos de Notificação dos municípios brasileiros. Utilizou-se modelo de regressão binomial negativo entre 2001 e 2015. Resultados: a análise das variações geográficas e temporais mostrou comportamentos heterogêneos dos três indicadores epidemiológicos de hanseníase. O aumento significativo desses foi associado à proporção de casos diagnosticados na Atenção Primária à Saúde $(p<0,001 ; p=0,003 ; p=0,015)$; já a proporção da cobertura populacional estimada por Estratégia Saúde da Família foi associada somente à redução significativa do indicador taxa de detecção entre menores de quinze anos $(\mathrm{p}=0,017)$. Conclusão: a interpretação simultânea dos principais indicadores epidemiológicos da hanseníase no Brasil reforçou a gravidade da situação e evidenciou que a satisfatória disponibilidade da ESF é insuficiente para o controle da doença.

Descritores: Hanseníase. Vigilância em Saúde Pública. Atenção Primária à Saúde.

Objective: to analyze the epidemiological situation of leprosy in Brazil and its association with the decentralization of control actions. Method: mixed ecological study with secondary data from the Sistema de Informação de Agravos de Notificação (Brazilian Information System on Notifiable Diseases) of Brazilian municipalities. A negative binomial regression model was used between 2001 and 2015. Results: The analysis of geographic and temporal variations showed heterogeneous behavior of the three epidemiological indicators of leprosy. The significant increase in these was associated with the proportion of cases diagnosed in Primary Health Care $(p<0.001 ; p=0.003 ; p=0.015)$; whereas the proportion of population coverage estimated by the Family Health Strategy (FHS) was associated only with the significant reduction in the indicator detection rate among children under fifteen $(p=0.017)$. Conclusion: the simultaneous interpretation of the main epidemiological indicators of leprosy in Brazil reinforced the gravity of the situation and evidenced that the satisfactory availability of the FHS is insufficient for the control of the disease.

Descriptors: Leprosy. Public Health Surveillance. Primary Health Care.

\footnotetext{
Enfermeira. Doutora em Enfermagem. Professora da Universidade Federal de Viçosa. Viçosa, Minas Gerais, Brasil. rayssa nr@yahoo.com.br. http://orcid.org/0000$0002-4772-4968$

Enfermeiro. Doutor em Enfermagem e Saúde Pública. Professor da Universidade de São Paulo. Ribeirão Preto, São Paulo, Brasil. http://orcid.org/0000-0003$4792-87 \mid 4$.

Enfermeiro. Doutor em Enfermagem. Professor da Universidade Federal de Minas Gerais. Belo Horizonte, Minas Gerais, Brasil. http://orcid.org/0000-000I$9043-3181$.
} 
Objetivo: analizar la situación epidemiológica de la lepra en Brasil y su asociación con la descentralización de las acciones de control. Método: estudio ecológico misto con datos secundarios del Sistema de Información de Agravamientos de Notificación de los municipios brasileños. Se utilizó un modelo de regresión binomial negativa entre 2001 y 2015. Resultados: el análisis de las variaciones geográficas y temporales mostró un comportamiento heterogéneo de los tres indicadores epidemiológicos de la lepra. El aumento significativo se asoció a la proporción de casos diagnosticados en Atención Primaria de Salud ( $p<0,001, p=0,003, p=0,015)$, mientras que la proporción de cobertura poblacional estimada por la Estrategia de Salud Familiar (ESF) se asoció únicamente a la reducción significativa de la tasa de detección del indicador entre los menores de quince años $(p=0,017)$. Conclusión: la interpretación simultánea de los principales indicadores epidemiológicos de la lepra en Brasil reforzó la gravedad de la situación y evidenció que la satisfactoria disponibilidad del ESF es insuficiente para el control de la enfermedad.

Descriptores: Lepra. Vigilancia en Salud Pública. Atención Primaria de Salud.

\section{Introdução}

A hanseníase permanece como um grave problema de saúde pública no mundo. Índia, Brasil e Indonésia são os países mais endêmicos, responsáveis por mais de $80 \%$ dos casos regis$\operatorname{trados}^{(1)}$. Nesses países, o quadro epidemiológico é ainda mais diversificado. No Brasil, aglomerados de risco concentram-se nas regiões Norte, Nordeste e Centro-Oeste, representando um taxa de detecção média de casos de 59,19 por 100 mil habitantes ${ }^{(2)}$. Para reverter esse quadro, estratégias vêm sendo adotadas. Dentre essas, destaca-se a descentralização das ações de controle da hanseníase (ACH).

O conceito de descentralização assumido na Reforma Sanitária brasileira tem um caráter político-administrativo, mas a legislação sobre o controle da hanseníase no Brasil assume a descentralização como a atenção prestada pelas unidades de Atenção Primária à Saúde (APS) na esfera municipal $^{(3)}$. A descentralização das ACH fortalece a política de controle da doença, ao facilitar o acesso ao diagnóstico e ao tratamento ${ }^{(4)}$. A APS representa o primeiro ponto de atenção do sistema de saúde, levando o atendimento o mais próximo possível do local onde as pessoas residem e trabalham. Contudo, a descentralização das ACH exige maiores progressos, como a expansão da cobertura da APS e a sustentabilidade das ações pelos profissionais ${ }^{(5)}$.

Com os Objetivos de Desenvolvimento Sustentável (ODS) para 2030, há um compromisso político para o fortalecimento da APS por meio da sua expansão e da oferta de ações de saúde ${ }^{(6)}$.
No entanto, a ausência de medição do desempenho da APS em responder às necessidades de saúde da população dificulta o alcance do compromisso político para o seu fortalecimento ${ }^{(7)}$. De uma perspectiva global, estudo realizado em 123 países demonstrou que o índice de cobertura de serviços à saúde não considerou as ações para as doenças tropicais negligenciadas ${ }^{(8)}$. A hanseníase enquadra-se nesse grupo e há anos tende a coexistir em áreas socialmente vulneráveis.

Assim, superar as desigualdades sociais exige reconhecer a saúde como um direito de cidadania e priorizar as necessidades de grupos específicos, segundo critérios de vulnerabilidade. No campo prático, isso significa aprimorar, principalmente nas áreas de alta endemicidade, diagnóstico precoce, tratamento oportuno, vigilância de contatos e a busca ativa de sintomáticos dermatoneurológicos.

Foi realizada pelos autores desta pesquisa uma revisão sistemática da literatura em 2019, ocasião em que não foram encontrados estudos que tivessem verificado como as áreas mais críticas em termos da carga da doença tinham se comportado a partir do processo de descentralização das ações de controle. Para a seleção das publicações, realizou-se a busca online no Portal Regional da Biblioteca Virtual em Saúde (BVS), Scopus e Web of Science. Foram incluídos artigos publicados nos idiomas português, inglês e espanhol.

A estratégia de busca utilizada na BVS foi: (tw:((hanseníase OR "Doença de Hansen" OR 
lepra OR leprosy))) AND (tw:(("Análise Espacial" OR "Análise Espaço-Temporal” OR "Análise por Conglomerados" OR conglomerados OR "Conglomerados Espaço-Temporais" OR "Análisis Espacial" OR "Análisis Espacio-Temporal" OR "Análisis por Conglomerados" OR "Agrupamiento Espacio-Temporal" OR "Spatial Analysis" OR "Spatio-Temporal Analysis" OR "Cluster Analysis" OR cluster OR "Space-Time Clustering"))) AND (tw:(("Atenção Primária à Saúde” OR "Atenção Básica à Saúde" OR descentralização OR "Atención Primaria de Salud" OR descentralización OR "Primary Health Care" OR decentralization))) AND (instance:"regional"); e na Scopus e Web of Science foi: Leprosy AND ("Spatial Analysis" OR "Spatio-Temporal Analysis" OR "Cluster Analysis" OR Cluster OR "Space-Time Clustering") AND ("Primary Health Care" OR Decentralization).

Esta pesquisa tem como objetivo analisar a situação epidemiológica da hanseníase no Brasil e sua associação com a descentralização das ações de controle.

\section{Método}

Trata-se de um estudo ecológico misto, que combina características de estudos de múltiplos grupos e de séries temporais. Neste tipo de estudo, pretende-se analisar associações entre as variáveis tendo em conta as suas mudanças ao longo do tempo e em vários locais. As orientações da iniciativa Strengthening the Reporting of Observational Studies in Epidemiology (Strobe) $^{(9)}$ foram seguidas para organizar o estudo a partir de seu desenho metodológico.

Foram consideradas como unidades territoriais de análise os aglomerados espaço-temporais identificados com base na taxa de detecção de casos novos de hanseníase residentes nos 5.565 municípios brasileiros definidos pelo censo demográfico 2010. Adotou-se a abordagem metodológica previamente relatada $^{(2)}$, incorporando, no entanto, o fator tempo. Nesse sentido, o interesse recaiu sobre a identificação de aglomerados que ocorreram no espaço e no tempo simultaneamente.

Essa abordagem consiste na identificação de aglomerados por meio de uma janela geográfica que se move com o centro das coordenadas em cada sede municipal. No caso dos aglomerados espaço-temporais, é utilizada uma janela cilíndrica, na qual a base representa o espaço e a altura o tempo (ano).

Os dados compreenderam os anos de 2001 a 2015. O período médio de incubação da hanseníase é de 2 a 5 anos, mas pode ser superior. Assim, é necessário analisar um período mais longo, para inferir o impacto do serviço na transmissão da doença. Todos os dados foram extraídos pelo próprio pesquisador em setembro de 2016.

A proporção da cobertura populacional estimada por Estratégia Saúde da Família (ESF) (Numerador: número de ESF multiplicado por 3.450 habitantes. Denominador: número de residentes no município) $)^{(10)}$ e a proporção de casos novos de hanseníase diagnosticados na APS (Numerador: casos novos residentes em determinado local e notificados na APS do município de residência. Denominador: casos novos notificados na atenção primária, secundária e terciária do município de residência) foram construídos para serem apresentadas como variáveis independentes e traduzirem a avaliação da descentralização das ACH para a APS.

Para compor o grupo das variáveis dependentes, três indicadores dispostos nas diretrizes para o controle da hanseníase do Ministério da Saúde foram selecionados ${ }^{(11)}$ : taxa de detecção anual de casos novos de hanseníase por 100 mil habitantes; taxa de detecção anual de casos novos de hanseníase na população de zero a 14 anos, por 100 mil habitantes; taxa de casos novos de hanseníase com grau 2 de incapacidade física no momento do diagnóstico por 100 mil habitantes.

O porte populacional e o ano de diagnóstico dos casos de hanseníase foram utilizados como variáveis controle no modelo estatístico. O processo de descentralização no Brasil é muito variado e resulta em grandes diferenças na cobertura e no acesso às ações de saúde ${ }^{(12)}$. As dimensões populacionais, por exemplo, refletem diferentes exigências sobre a política local em termos de complexidade e de escala dos serviços ${ }^{(13)}$. Além disso, os dados epidemiológicos da hanseníase são extremamente sensíveis à capacidade operacional dos serviços de saúde, podendo variar de ano para ano ${ }^{(14)}$. 
As informações dos casos de hanseníase foram retiradas da base de dados do Sistema de Informação de Agravos de Notificação (Sinan), disponibilizadas pelo Ministério da Saúde por meio do Sistema Eletrônico do Serviço de Informações ao Cidadão (e-SIC). O número de habitantes por município foi obtido no censo populacional e nas estimativas intercensitárias (metodologia adotada para estimar o número de pessoas nos municípios entre dois censos demográficos consecutivos) da base de dados do Instituto Brasileiro de Geografia e Estatísticas (IBGE). A cobertura populacional estimada por ESF foi retirada da base de dados do Departamento de Atenção Básica da Secretaria de Atenção à Saúde do Ministério da Saúde. Para a identificação dos estabelecimentos de saúde onde os casos de hanseníase foram diagnosticados, foi realizada uma consulta ao Cadastro Nacional de Estabelecimentos de Saúde (CNES), com base nos códigos da unidade notificadora apresentados no Sinan.

Para construção e cálculo dos indicadores, foram utilizados o aplicativo de acesso livre Tab para Windows (TabWin) (versão 3.2) e o software Microsoft Office Excel (versão 2016). Na sequência, os dados foram exportados para o software R (versão 3.5.1) para as análises.

O modelo de efeito misto ${ }^{(15)}$ foi utilizado. Inicialmente, foram verificadas as variações geográficas e temporais das variáveis dependentes por aglomerado previamente identificado (efeito aleatório). Em seguida, foi verificada a relação entre as variáveis dependentes e independentes, estimando-se parâmetros específicos da população em estudo, isto é, os mesmos para todos os aglomerados (efeito fixo). Para a modelagem das variáveis, foi utilizado o modelo de regressão binomial negativo ${ }^{(16)}$.

Com o tempo centrado em 2008, o coeficiente $\beta 0$ (intercepto) significa o valor médio esperado para a variável resposta de interesse no ano de 2008 para todos os aglomerados, enquanto $\operatorname{Exp}(\alpha j)$ fornece o quanto o j-ésimo aglomerado está acima ou abaixo da média geral da variável resposta de interesse. O coeficiente $\beta_{1}$ fornece, em média, a tendência da variável resposta de interesse a cada um ano acrescido, enquanto que $\operatorname{Exp}(\beta 1+\mu j)$ fornece, em média, a tendência para a variável resposta de interesse ao longo do tempo para o j-ésimo aglomerado. O coeficiente $\beta_{2}$ representa o efeito de cada variável independente sobre a variável dependente. Este modelo é conhecido como modelo de efeito misto, pois se tem o efeito fixo $(\beta 0, \beta 1$, $\beta 2$ ) e o efeito aleatório $\left(\alpha_{j} e \mu_{j}\right)$. A notação j significa $\mathrm{j}=1,2, \ldots, 15$ (aglomerado).

No modelo ajustado, foi aplicado o método Backward e adotado um nível de 5\% de significância. Para verificar a presença de multicolinearidade entre as variáveis independentes, foi utilizada a estatística Fator de Inflação da Variância (VIF), sendo retiradas do modelo as variáveis com VIF superior a dez ${ }^{(17)}$.

Este estudo foi realizado atendendo às determinações da Resolução no 466/2012 do Conselho Nacional de Saúde e aprovado pelo Comitê de Ética em Pesquisa (CEP) da Universidade Federal de Minas Gerais, Parecer no 2.125.179, de 22 de junho de 2017.

\section{Resultados}

Os quinze aglomerados identificados compreenderam 319.581 (52,77\%) casos de hanseníase. A taxa média de detecção na população total foi de 58,48 por 100 mil habitantes, enquanto para os indicadores taxa de detecção entre os menores de quinze anos de idade e taxa de grau 2 de incapacidade física no momento do diagnóstico, os valores foram de 14,39 por 100 mil e 3,19 por 100 mil habitantes, respectivamente. Para todos os indicadores analisados houve aglomerados que sobrepuseram as médias do período (Figura 1 e Tabela 1). 
Figura 1 - Taxa média e tendência de detecção dos indicadores epidemiológicos de hanseníase por aglomerado. Brasil - 2001-2015 (N=319.581)

Taxa média de detecção de hanseníase na população total

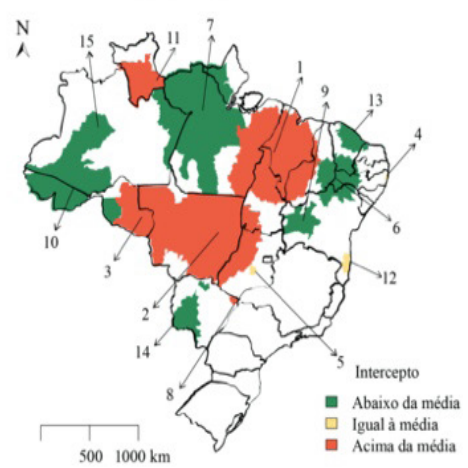

Taxa média de detecção de hanseníase na população $<15$ anos

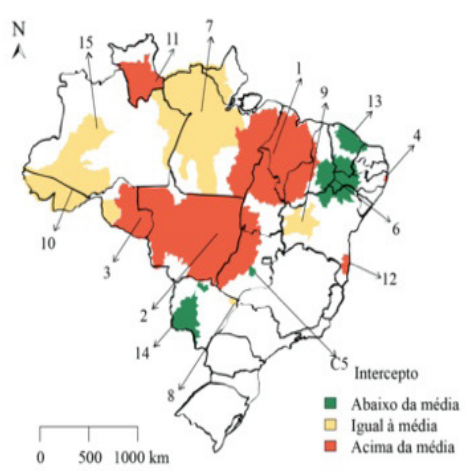

Taxa média de deteção de casos de hanseníase com grau 2 de incapacidade fisica no diagnóstico

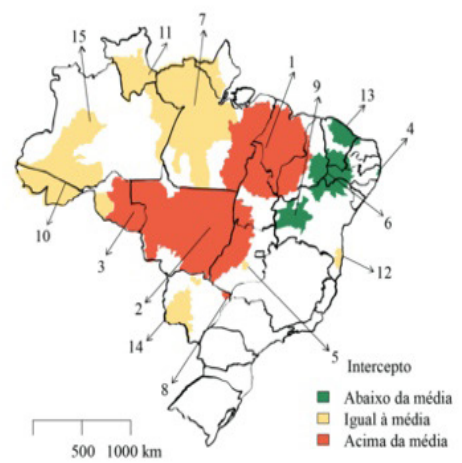

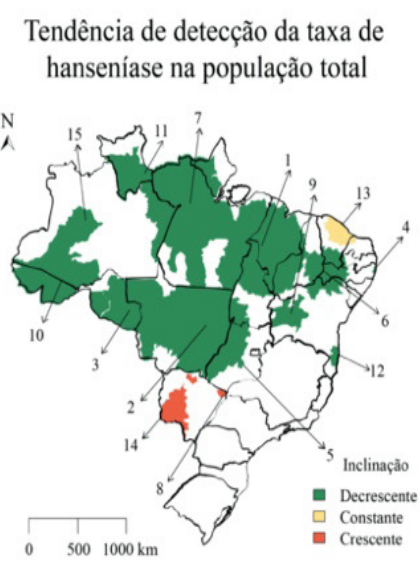

Tendência de detecção de hanseníase na população $<15$ anos

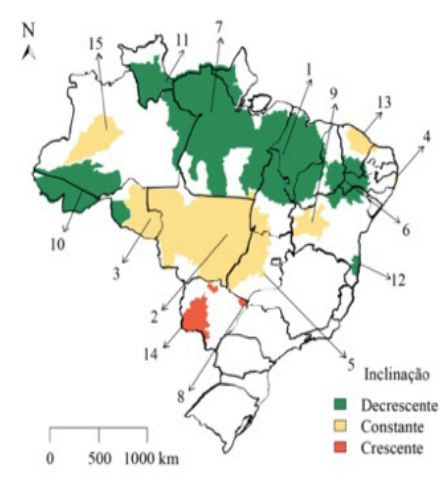

Tendência de detecção de casos de hanseníase com grau 2 de incapacidade fisica no diagnóstico

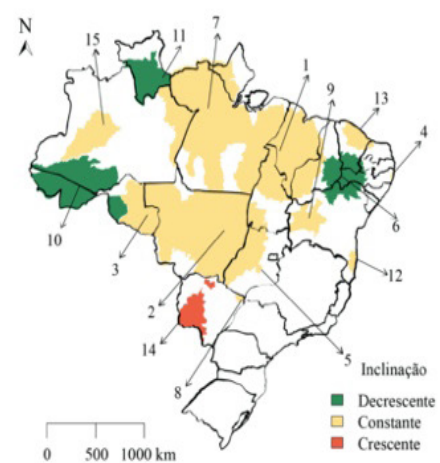

Fonte: Elaboração própria.

Nota: Taxas médias e tendências estimadas pelo modelo de efeito misto com distribuição binomial negativo. 
Tabela 1 - Taxa média dos indicadores epidemiológicos de hanseníase por aglomerado. Brasil - 2001-2015. (N=319.581)

\begin{tabular}{l|c|c|c|c|c|c}
\hline \multirow{2}{*}{ Aglomerado } & \multicolumn{2}{|c|}{ Taxa na população total } & \multicolumn{2}{c|}{$\begin{array}{c}\text { Taxa em menores de } \\
\text { quinze anos }\end{array}$} & \multicolumn{2}{c}{$\begin{array}{c}\text { Taxa de grau 2 de } \\
\text { incapacidade fisica }\end{array}$} \\
\cline { 2 - 7 } & $\begin{array}{c}\text { Exponencial } \\
\text { (aj) }\end{array}$ & $\begin{array}{c}\text { Intervalo de } \\
\text { Confiança } \\
\mathbf{9 5 \%}\end{array}$ & $\begin{array}{c}\text { Exponencial } \\
(\mathbf{\alpha} \mathbf{j})\end{array}$ & $\begin{array}{c}\text { Intervalo de } \\
\text { Confiança } \\
\mathbf{9 5 \%}\end{array}$ & $\begin{array}{c}\text { Exponencial } \\
\text { (aj) }\end{array}$ & $\begin{array}{c}\text { Intervalo de } \\
\text { Confiança } \\
\mathbf{9 5 \%}\end{array}$ \\
\hline 1 & 1,371 & {$[1,343 ; 1,400]$} & 1,779 & {$[1,713 ; 1,848]$} & 1,410 & {$[1,350 ; 1,473]$} \\
2 & 1,920 & {$[1,867 ; 1,975]$} & 1,908 & {$[1,797 ; 2,025]$} & 1,494 & {$[1,399 ; 1,596]$} \\
3 & 1,601 & {$[1,528 ; 1,679]$} & 1,395 & {$[1,269 ; 1,534]$} & 1,492 & {$[1,352 ; 1,646]$} \\
4 & 0,930 & {$[0,845 ; 1,024]$} & 1,527 & {$[1,315 ; 1,773]$} & 0,687 & {$[0,585 ; 0,807]$} \\
5 & 1,068 & {$[0,969 ; 1,177]$} & 0,644 & {$[0,526 ; 0,788]$} & 0,922 & {$[0,763 ; 1,112]$} \\
6 & 0,786 & {$[0,757 ; 0,815]$} & 0,770 & {$[0,711 ; 0,833]$} & 0,796 & {$[0,732 ; 0,866]$} \\
7 & 0,922 & {$[0,867 ; 0,980]$} & 0,904 & {$[0,810 ; 1,009]$} & 1,055 & {$[0,936 ; 1,188]$} \\
8 & 1,780 & {$[1,304 ; 2,429]$} & 1,237 & {$[0,725 ; 2,113]$} & 1,803 & {$[1,156 ; 2,811]$} \\
9 & 0,861 & {$[0,788 ; 0,941]$} & 0,848 & {$[0,711 ; 1,011]$} & 0,526 & {$[0,423 ; 0,656]$} \\
10 & 0,880 & {$[0,818 ; 0,946]$} & 1,011 & {$[0,882 ; 1,158]$} & 0,999 & {$[0,856 ; 1,166]$} \\
11 & 1,240 & {$[1,087 ; 1,415]$} & 1,391 & {$[1,090 ; 1,776]$} & 1,258 & {$[0,965 ; 1,639]$} \\
12 & 0,959 & {$[0,867 ; 1,059]$} & 1,621 & {$[1,363 ; 1,929]$} & 0,904 & {$[0,736 ; 1,111]$} \\
13 & 0,435 & {$[0,415 ; 0,457]$} & 0,341 & {$[0,306 ; 0,379]$} & 0,588 & {$[0,532 ; 0,651]$} \\
14 & 0,734 & {$[0,667 ; 0,807]$} & 0,414 & {$[0,324 ; 0,529]$} & 1,029 & {$[0,846 ; 1,253]$} \\
15 & 0,644 & {$[0,551 ; 0,753]$} & 0,915 & {$[0,699 ; 1,198]$} & 0,916 & {$[0,679 ; 1,236]$} \\
\hline
\end{tabular}

Fonte: Elaboração própria.

Nota: Intervalo de Confiança (IC) de 95\% para as taxas de cada aglomerado estimado pelo modelo de efeito misto com distribuição binomial negativo.

Quanto à análise das séries históricas, chama a atenção a presença de aglomerados com tendências crescentes e constantes (Figura 1 e Tabela 2).

Tabela 2 - Tendência dos indicadores epidemiológicos de hanseníase por aglomerado. Brasil - 20012015. $(\mathrm{N}=319.581)$

\begin{tabular}{|c|c|c|c|c|c|c|}
\hline \multirow[b]{2}{*}{ Aglomerado } & \multicolumn{2}{|c|}{$\begin{array}{c}\text { Taxa na população } \\
\text { total }\end{array}$} & \multicolumn{2}{|c|}{$\begin{array}{c}\text { Taxa em menores de } \\
\text { quinze anos }\end{array}$} & \multicolumn{2}{|c|}{$\begin{array}{l}\text { Taxa de grau } 2 \text { de } \\
\text { incapacidade física }\end{array}$} \\
\hline & $\begin{array}{c}\text { Exponencial } \\
(\beta 1+\boldsymbol{\mu} j)\end{array}$ & \begin{tabular}{|c|}
$\begin{array}{c}\text { Intervalo de } \\
\text { Confiança } \\
95 \%\end{array}$ \\
\end{tabular} & $\begin{array}{l}\text { Exponencial } \\
\quad(\beta 1+\boldsymbol{\mu} j)\end{array}$ & $\begin{array}{c}\text { Intervalo de } \\
\text { Confiança } \\
95 \% \\
\end{array}$ & $\begin{array}{c}\text { Exponencial } \\
(\beta 1+\mu j)\end{array}$ & $\begin{array}{c}\text { Intervalo de } \\
\text { Confiança } \\
95 \% \\
\end{array}$ \\
\hline 1 & 0,948 & {$[0,923 ; 0,975]$} & 0,958 & {$[0,924 ; 0,993]$} & 0,975 & {$[0,949 ; 1,002]$} \\
\hline 2 & 0,965 & {$[0,939 ; 0,992]$} & 0,970 & {$[0,934 ; 1,008]$} & 1,002 & {$[0,974 ; 1,032]$} \\
\hline 3 & 0,960 & {$[0,932 ; 0,989]$} & 0,969 & {$[0,929 ; 1,010]$} & 0,992 & {$[0,959 ; 1,025]$} \\
\hline 4 & 0,963 & {$[0,929 ; 0,997]$} & 0,988 & {$[0,941 ; 1,038]$} & 0,966 & {$[0,925 ; 1,008]$} \\
\hline 5 & 0,941 & {$[0,909 ; 0,974]$} & 0,955 & {$[0,903 ; 1,011]$} & 0,987 & {$[0,942 ; 1,034]$} \\
\hline 6 & 0,961 & {$[0,934 ; 0,989]$} & 0,960 & {$[0,922 ; 0,999]$} & 0,963 & {$[0,933 ; 0,994]$} \\
\hline 7 & 0,945 & {$[0,916 ; 0,974]$} & 0,952 & {$[0,911 ; 0,994]$} & 0,997 & {$[0,961 ; 1,033]$} \\
\hline 8 & 1,097 & {$[1,025 ; 1,174]$} & 1,135 & {$[1,025 ; 1,257]$} & 1,054 & {$[0,977 ; 1,137]$} \\
\hline 9 & 0,965 & {$[0,933 ; 0,998]$} & 0,995 & {$[0,943 ; 1,050]$} & 0,998 & {$[0,948 ; 1,050]$} \\
\hline 10 & 0,913 & {$[0,885 ; 0,943]$} & 0,922 & {$[0,879 ; 0,967]$} & 0,948 & {$[0,909 ; 0,988]$} \\
\hline 11 & 0,920 & {$[0,883 ; 0,958]$} & 0,909 & {$[0,852 ; 0,970]$} & 0,926 & {$[0,874 ; 0,982]$} \\
\hline 12 & 0,932 & {$[0,899 ; 0,966]$} & 0,921 & {$[0,873 ; 0,972]$} & 0,995 & {$[0,947 ; 1,046]$} \\
\hline 13 & 0,981 & {$[0,953 ; 1,010]$} & 1,007 & {$[0,965 ; 1,052]$} & 0,980 & {$[0,947 ; 1,013]$} \\
\hline 14 & 1,079 & {$[1,042 ; 1,117]$} & 1,114 & {$[1,045 ; 1,187]$} & 1,100 & {$[1,048 ; 1,153]$} \\
\hline 15 & 0,935 & {$[0,895 ; 0,978]$} & 0,942 & {$[0,880 ; 1,008]$} & 0,979 & {$[0,920 ; 1,041]$} \\
\hline
\end{tabular}

Fonte: Elaboração própria.

Nota: Intervalo de Confiança (IC) de 95\% para as taxas de cada aglomerado estimado pelo modelo de efeito misto com distribuição binomial negativo. 
Em relação à descentralização das ações de controle, os resultados evidenciam que a proporção da cobertura populacional estimada pela ESF foi estatisticamente significativa somente para a redução da taxa de detecção entre os menores de 15 anos. Já para a proporção de casos diagnosticados na APS, associação estatisticamente significativa foi observada em todos os indicadores analisados, com aumento das taxas (Tabela 3).

Tabela 3 - Relação entre o diagnóstico da Atenção Primária à Saúde e a cobertura da Estratégia Saúde da Família com os indicadores epidemiológicos de hanseníase nos aglomerados (modelo inicial e modelo final). Brasil - 2001-2015. ( $N=319.581)$

(continua)

\begin{tabular}{|c|c|c|c|c|c|}
\hline \multirow[b]{2}{*}{ Variáveis } & \multicolumn{5}{|c|}{ Modelo inicial } \\
\hline & $\begin{array}{c}\text { Coeficiente } \\
\text { da regressão } \\
(\beta)\end{array}$ & $\begin{array}{c}\text { Erro Padrão } \\
\text { do coeficiente } \\
(\beta)\end{array}$ & $\begin{array}{c}\text { Exponencial } \\
(\beta)\end{array}$ & $\begin{array}{c}\text { Intervalo de } \\
\text { Confiança } \\
95 \%\end{array}$ & Valor-p \\
\hline $\begin{array}{l}\text { Taxa de hanseníase na } \\
\text { população total }\end{array}$ & $-7,397$ & 0,108 & - & - & $<0,001$ \\
\hline $\begin{array}{l}\text { Casos diagnosticados } \\
\text { na Atenção Primária à } \\
\text { Saúde/10 }\end{array}$ & 0,011 & 0,002 & 1,011 & {$[1,007 ; 1,015]$} & $<0,001$ \\
\hline $\begin{array}{l}\text { Cobertura da Estratégia } \\
\text { Saúde da Família/10 }\end{array}$ & $-0,003$ & 0,003 & 0,997 & {$[0,992 ; 1,002]$} & 0,217 \\
\hline $\begin{array}{l}\text { Fator de Inflação da } \\
\text { Variância-Máximo }\end{array}$ & & & 3,11 & & \\
\hline $\begin{array}{l}\text { Taxa de hanseníase na } \\
\text { população }<15 \text { anos }\end{array}$ & $-8,914$ & 0,154 & - & - & $<0,001$ \\
\hline $\begin{array}{l}\text { Casos diagnosticados } \\
\text { na Atenção Primária à } \\
\text { Saúde/10 }\end{array}$ & 0,011 & 0,004 & 1,011 & {$[1,004 ; 1,018]$} & 0,003 \\
\hline $\begin{array}{l}\text { Cobertura da Estratégia } \\
\text { Saúde da Família/10 }\end{array}$ & $-0,012$ & 0,005 & 0,988 & {$[0,979 ; 0,998]$} & 0,017 \\
\hline $\begin{array}{l}\text { Fator de Inflação da } \\
\text { Variância-Máximo }\end{array}$ & & & 5,08 & & \\
\hline $\begin{array}{l}\text { Taxa de hanseníase com } \\
\text { grau } 2 \text { de incapacidade } \\
\text { física }\end{array}$ & $-7,397$ & 0,108 & - & - & $<0,001$ \\
\hline $\begin{array}{l}\text { Casos diagnosticados } \\
\text { na Atenção Primária à } \\
\text { Saúde/10 }\end{array}$ & 0,011 & 0,002 & 1,011 & {$[1,007 ; 1,015]$} & $<0,001$ \\
\hline $\begin{array}{l}\text { Cobertura da Estratégia } \\
\text { Saúde da Família/10 }\end{array}$ & $-0,003$ & 0,003 & 0,997 & {$[0,992 ; 1,002]$} & 0,217 \\
\hline $\begin{array}{l}\text { Fator de Inflação da } \\
\text { Variância-Máximo }\end{array}$ & & & 6,36 & & \\
\hline
\end{tabular}

\begin{tabular}{l|c|c|c|c|c}
\hline \hline & \multicolumn{4}{|c}{ Modelo final } \\
\cline { 2 - 6 } Variáveis & $\begin{array}{c}\text { Coeficiente } \\
\text { da } \\
\text { regressão } \\
(\beta)\end{array}$ & $\begin{array}{c}\text { Erro Padrão } \\
\text { do coeficiente } \\
(\beta)\end{array}$ & $\begin{array}{c}\text { Exponencial } \\
(\beta)\end{array}$ & $\begin{array}{c}\text { Intervalo de } \\
\text { Confiança } \\
\mathbf{9 5 \%}\end{array}$ & Valor-p \\
\hline $\begin{array}{l}\text { Taxa de hanseníase na } \\
\text { população total }\end{array}$ & $-7,424$ & 0,105 & - & - & $<0,001$ \\
$\begin{array}{l}\text { Casos diagnosticados } \\
\text { na Atenção Primária à }\end{array}$ & 0,011 & 0,002 & 1,011 & {$[1,007 ; 1,015]$} & $<0,001$ \\
Saúde/10 & & & & & \\
Cobertura da Estratégia \\
Saúde da Família/10 \\
Fator de Inflação da \\
Variância - Máximo
\end{tabular}


Tabela 3 - Relação entre o diagnóstico da Atenção Primária à Saúde e a cobertura da Estratégia Saúde da Família com os indicadores epidemiológicos de hanseníase nos aglomerados (modelo inicial e modelo final). Brasil - 2001-2015. (N=319.581)

(conclusão)

\begin{tabular}{|c|c|c|c|c|c|}
\hline \multirow[b]{2}{*}{ Variáveis } & \multicolumn{5}{|c|}{ Modelo final } \\
\hline & \begin{tabular}{|c|}
$\begin{array}{c}\text { Coeficiente } \\
\text { da regressão } \\
(\beta)\end{array}$ \\
\end{tabular} & \begin{tabular}{|c|}
$\begin{array}{c}\text { Erro Padrão } \\
\text { do coeficiente } \\
(\beta)\end{array}$ \\
\end{tabular} & $\begin{array}{c}\text { Exponencial } \\
(\beta)\end{array}$ & $\begin{array}{c}\text { Intervalo de } \\
\text { Confiança } \\
95 \% \\
\end{array}$ & Valor-p \\
\hline \multicolumn{6}{|l|}{$\begin{array}{l}\text { Taxa de hanseníase na } \\
\text { população }<15 \text { anos }\end{array}$} \\
\hline $\begin{array}{l}\text { Casos diagnosticados } \\
\text { na Atenção Primária à } \\
\text { Saúde/10 }\end{array}$ & - & - & - & - & - \\
\hline Cobertura da Estratégia & - & - & - & - & - \\
\hline $\begin{array}{l}\text { Saúde da Família/10 } \\
\text { Fator de Inflação da } \\
\text { Variância - Máximo }\end{array}$ & & & - & & \\
\hline $\begin{array}{l}\text { Taxa de hanseníase com } \\
\text { grau } 2 \text { de incapacidade } \\
\text { física }\end{array}$ & $-10,522$ & 0,101 & - & - & $<0,001$ \\
\hline $\begin{array}{l}\text { Casos diagnosticados } \\
\text { na Atenção Primária à } \\
\text { Saúde/10 } \\
\text { Cobertura da Estratégia } \\
\text { Saúde da Família/10 }\end{array}$ & 0,010 & 0,004 & 1,010 & {$[1,002 ; 1,017]$} & 0,015 \\
\hline $\begin{array}{l}\text { Fator de Inflação da } \\
\text { Variância - Máximo }\end{array}$ & & & 1,00 & & \\
\hline Fonte: Elaboração própria. & & & & & \\
\hline
\end{tabular}

\section{Discussão}

A permanência da hiperendemicidade das taxas de detecção na população total (>40,00/100 mil habitantes) e entre os menores de quinze anos $(\geq 10,00 / 100 \text { mil habitantes })^{(11)}$ sugere falhas dos serviços de saúde. Quando a detecção é parte das ações das equipes, a taxa na população total aumenta consideravelmente, entretanto, nos anos seguintes, tende a diminuir ${ }^{(18)}$. Ainda, os dados apresentam indícios de existência de doença recente e focos ativos de transmissão, uma vez que a velocidade de queda da taxa de detecção entre os menores de quinze anos não foi maior do que da taxa de detecção na população total em todos os aglomerados.

Outro agravante é a tendência crescente ou mesmo constante da taxa de grau 2 de incapacidade física no momento do diagnóstico. O grau de incapacidade física é definido pela avaliação neurológica dos olhos, mãos e pés, e pode ser classificado em 0,1 e 2 , sendo o último responsável por maiores limitações ${ }^{(11)}$. Esse indicador sinaliza a baixa eficiência da detecção precoce pela equipe de saúde, o que contribui para a situação endêmica oculta.

A atual estratégia global de combate à hanseníase da Organização Mundial da Saúde (OMS) enfatiza, dentre outras ações, a promoção da detecção precoce, isto é, antes do surgimento de incapacidades, com ênfase especial nas crianças ${ }^{(1)}$. No entanto, o que se observa é que o país ainda não alcançou um dos princípios-chave dessa estratégia, pois há ocorrência de incapacidades em crianças.

A despeito da importância do fator operacional e com o objetivo de entender os motivos que levam à agenda inconclusa no controle da hanseníase no Brasil, os principais indicadores epidemiológicos propostos pelo Programa 
Nacional de Controle da Hanseníase foram analisados à luz da cobertura da ESF e do diagnóstico de casos na APS. Acreditava-se que ambas as variáveis fossem igualmente importantes para o controle da doença, no entanto os resultados da presente pesquisa refutam essa hipótese. A expansão da ESF foi associada somente à redução da taxa de detecção entre os menores de 15 anos.

No Brasil, a principal estratégia para a descentralização das ACH para APS ocorre por meio da ESF, composta por uma equipe multiprofissional. Cada ESF é responsável por uma população definida, dentro de uma área. Ao se responsabilizarem pela saúde da população adscrita, os profissionais inseridos na ESF ampliam a prática do modelo biomédico, promovendo também a melhoria das condições de vida, por meio de ações intersetoriais ${ }^{(19)}$. A oferta dessas ações tem papel importante para a redução da transmissão da hanseníase.

Nesse sentido, é provável que a queda da detecção entre os menores de quinze anos esteja relacionada às ações ampliadas da ESF e não à capacidade de manejo dos casos pelos profissionais, pois a cobertura da ESF não impactou na detecção de casos na população total. A execução do teste de sensibilidade em crianças é mais difícil, pois muitas crianças não compreendem as instruções. Além disso, nas crianças, as características clínicas apresentam-se, muitas vezes, como dermatite crônica inespecífica $^{(20)}$.

O desafio atual compreende a realização de ações pelos profissionais de saúde, como, por exemplo, o diagnóstico da hanseníase. Embora esse diagnóstico seja uma atribuição médica, a sua suspeição pode ser realizada pelo enfermeiro. Estudo recente apontou o interesse em valer-se dos enfermeiros para expandir a capacidade da força de trabalho na APS ${ }^{(21)}$. As consultas mais longas, o número de retornos assistidos e o vínculo são exemplos que demonstram a capacidade de o enfermeiro fornecer qualidade de atendimento igual ou possivelmente melhor que a do profissional médico ${ }^{(21)}$.

Assim, o aumento da detecção de casos na população total associado à proporção de casos diagnosticados na APS pode ser resultado da situação operacional, isso é, da capacidade de os profissionais identificarem os sinais clínicos da hanseníase e não de base epidemiológica.

O impacto da proporção de casos diagnosticados na APS sobre a taxa de detecção em menores de quinze anos pode ser explicado pelas ações dos profissionais de saúde, particularmente pelas triagens direcionadas ao ambiente escolar. Estudo realizado no Brasil apontou que abordagens em larga escala com escolares residentes em municípios hiperendêmicos contribuíram para a elevação das taxas de detecção, superando os registros oficiais ${ }^{(22)}$.

A presença de profissionais sensibilizados/treinados na realização das $\mathrm{ACH}$ reflete-se também na taxa de grau 2 de incapacidade física no diagnóstico. O aumento desse indicador pode significar que o serviço está apenas desenvolvendo ações sobre o estoque de casos diagnosticados tardiamente ${ }^{(23)}$. No entanto, não se pode descartar a ocorrência de fragilidades na detecção precoce. O diagnóstico da hanseníase é essencialmente clínico. É comum ser subdiagnosticada, pois seus sinais são confundidos com os de outras doenças de pele relativamente inofensivas e as alterações acabam sendo ignoradas até por especialistas ${ }^{(24)}$.

Os resultados da presente pesquisa sugerem que o Brasil investigue mais a fundo se o progresso em direção à expansão da ESF está realmente priorizando as pessoas menos favorecidas. Ao focar no manejo de uma doença que atinge principalmente comunidades pobres, o indicador "proporção de casos de hanseníase diagnosticados na APS" forneceu uma maneira simples de avaliar a equidade na cobertura das $\mathrm{ACH}$.

Diante dessas considerações, evidencia-se que a sustentabilidade das ACH vai além da expansão da ESF. Os desafios para o aprimoramento desse ponto de atenção estão condicionados a fatores complexos e que exigem maiores esforços político-institucionais. É preciso superar: a insuficiência dos conteúdos curriculares sobre a hanseníase; a racionalidade biomédica no processo de trabalho; a rotatividade dos profissionais; as capacitações que, muitas 
vezes, não resultam em equipes confiantes para diagnosticar ou iniciar o tratamento; a centralidade da atenção ao indivíduo doente e não à família/ao coletivo; e a falta de priorização da hanseníase nas políticas de saúde.

Para estudos futuros, seria oportuno o maior detalhamento dos fatores organizacionais dos serviços inseridos nos aglomerados, incorporando a abordagem direta aos profissionais e aos usuários. Esses dados poderão fortalecer os achados da presente pesquisa e contribuir para o enfrentamento de dificuldades pontuais.

Por fim, é importante ressaltar que o CNES não leva em consideração as diferentes modalidades de APS, o que impossibilitou o cálculo do indicador "proporção de casos novos de hanseníase diagnosticados na ESF". Contudo, tal limitação não comprometeu as análises, pois, dentre as equipes vinculadas a estabelecimentos de saúde da APS, a ESF é responsável por mais de $86 \%$ da cobertura estimada ${ }^{(10)}$.

Entre as limitações deste estudo, cabe destacar que a utilização de dados secundários pode frequentemente resultar em inconsistências e não completitude. Apesar disso, os indicadores calculados com base no Sinan nacional foram confrontados com as publicações do Ministério da Saúde, fortalecendo a base de evidências do estudo. Outra limitação refere-se à mudança nos critérios de definição de caso com grau 2 de incapacidade física, que teve efeito nas notificações realizadas em $2007^{(25)}$. Assim, os dados de 2007 foram excluídos deste estudo.

\section{Conclusão}

Conclui-se que a interpretação simultânea dos principais indicadores epidemiológicos do Programa Nacional de Controle da Hanseníase reforça a gravidade da situação no país e que, embora a disponibilidade da ESF tenha apresentado resultados satisfatórios, ela sozinha é insuficiente para o controle da doença. A condição identificada como fundamental para atingir os parâmetros propostos pelo Ministério da Saúde é o aumento da oferta de ações de controle da hanseníase.
Espera-se que os resultados apresentados neste estudo subsidiem as políticas direcionadas à melhoria da qualidade da APS e promovam a ampliação equitativa do acesso e da utilização dos serviços.

\section{Colaborações:}

1 - concepção, projeto, análise e interpretação dos dados: Rayssa Nogueira Rodrigues;

2 - redação do artigo e revisão crítica relevante do conteúdo intelectual: Rayssa Nogueira Rodrigues, Ricardo Alexandre Arcêncio e Francisco Carlos Félix Lana;

3 - aprovação final da versão a ser publicada: Rayssa Nogueira Rodrigues, Ricardo Alexandre Arcêncio e Francisco Carlos Félix Lana.

\section{Referências}

1. World Health Organization. Global Leprosy Strategy 2016-2020: Accelerating towards a leprosy-free world [Internet] Genebra; 2016 [cited 2020 Jan 10]. Available from: https://apps.who.int/iris/bitstream/handle/10665/ 208824/9789290225096_en.pdf?sequence= 14\&isAllowed $=\mathrm{y}$

2. Rodrigues RN, Leano HAM, Bueno IC, Araújo KMFA, Lana FCF. High-risk areas of leprosy in Brazil between 2001-2015. Rev Bras Enferm. 2020 apr;73(3):e20180583. DOI: 10.1590/0034-7167-2018-0583

3. Brasil. Ministério da Saúde. Portaria no 1.073, de 26 de setembro de 2000. Expede as instruções normativas destinadas a orientar o desenvolvimento das ações de controle e de eliminação da hanseníase em todo o Território Nacional, com ênfase na atenção básica de saúde, as quais regulamentam as diretrizes estabelecidas pela Portaria no 816, de 26 de julho de 2000. Brasília (DF); 2000. Diário Oficial da União. Brasília (DF); 200027 set; Seção 1;(188):18-22.

4. Chudasama RK, Lakkad SG, Patel UV, Sheth A, Thakkar D, Rangoonwala M. Evaluation of national leprosy eradication program after integration into general health system in Rajkot District, Gujarat from 2003 to 2014. Indian J Dermatol. 2016 jan-feb;61(1):57-62. DOI: 10.4103/0019-5154.174027 
5. Macinko J, Harris MJ, Rocha MG. Brazil's national program for improving primary care access and quality (PMAQ): fulfilling the potential of the world's largest Payment for performance system in primary care. J Ambul Care Manage. 2017 apr-jun;40(Suppl 2):S4-S11. DOI: 10.1097/ JAC.0000000000000189

6. Kieny MP, Bekedam H, Dovlo D, Fitzgerald J, Habicht J, Harrison G, et al. Strengthening health systems for universal health coverage and sustainable development. Bull World Health Organ. 2017 Jul;95(7):537-9. DOI: 10.2471/BLT. 16.187476

7. Barbazza E, Kringos D, Kruse I, Klazinga NS, Tello JE. Creating performance intelligence for primary health care strengthening in Europe. BMC Health Serv Res. 2019 dec 27;19(1):1006. DOI: 10.1186/s12913-019-4853-Z

8. Fitzpatrick C, Bangert M, Mbabazi PS, Mikhailov A, Zouré H, Rebollo MP, et al. Monitoring equity in universal health coverage with essential services for neglected tropical diseases: an analysis of data reported for five diseases in 123 countries over 9 years. Lancet Glob Health. 2018 jul;6(9):e980-8. DOI: 10.1016/S2214-109X(18)30307-3

9. Von Elm E, Altman DG, Egger M, Pocock SJ, Gøtzsche PC, Vandenbroucke JP, et al. Strengthening the reporting of observational Studies in epidemiology (STROBE) statement: guidelines for reporting observational studies. BMJ. 2007 Oct;335(7624):806-8. DOI: 10.1136/ bmj.39335.541782.AD

10. Brasil. Ministério da Saúde. Informação e Gestão da Atenção Básica. Cobertura da Atenção Básica [Internet]. Brasília (DF); 2020 [cited 2020 Jan 15]. Available from: https://bit.ly/2VwmnPX

11. Brasil. Ministério da Saúde. Secretaria de Vigilância em Saúde. Departamento de Vigilância das Doenças Transmissíveis. Diretrizes para vigilância, atenção e eliminação da hanseníase como problema de saúde pública: manual técnico-operacional [Internet]. Brasília (DF); 2016 [cited 2020 Jan 10]. Available from: https:// portalarquivos2.saude.gov.br/images/pdf/2016/ fevereiro/04/diretrizes-eliminacao-hanseniase4fev16-web.pdf

12. Malta DC, Santos MAS, Stopa SR, Vieira JEB, Melo EA, Reis AAC. Family Health Strategy coverage in Brazil, according to the National Health Survey, 2013. Cien Saude Colet.
2016 Feb;21(2):327-38. DOI: 10.1590/141381232015212.23602015

13. Araújo CEL, Gonçalves GQ, Machado JA. Os municípios brasileiros e os gastos próprios com saúde: algumas associações. Cienc saúde coletiva. 2017 mar;22(3):953-63. DOI: 10.1590/ 1413-81232017223.15542016

14. Rodrigues RN, Niitsuma ENA, Bueno IC, Baquero OS, Jardim CCG, Lana FCF. Hanseníase e vulnerabilidade da saúde em Belo Horizonte, Minas Gerais. Rev Min Enferm. 2017;21:e-997. DOI: $10.5935 / 1415-2762.20170007$

15. Pinheiro JC, Bates DM. Mixed-Effects models in S and S-PLUS. New York: Springer; 2000.

16. Ver Hoef JM, Boveng PL. Quasi-poisson vs. negative binomial regression: how should we model overdispersed count data? Ecology. 2007 nov;88(11):2766-72. DOI: 10.1890/07-0043.1

17. Montgomery DC, Peck EA, Vining GG. Introduction to linear regression analysis. 5th ed. New York: John Wiley \& Sons; 2012.

18. Gomes FBFF, Lana FCF, Oliveira RC, Rodrigues RN. Indicadores da hanseníase no estado de Minas Gerais e sua relação com o índice de desenvolvimento humano municipal e a cobertura da Estratégia da Saúde da Família. Rev Min Enferm. 2017;21:e-1063. DOI:10.5935/ $1415-2762.20170073$

19. Bezerra IMP, Sorpreso ICE. Concepts and movements in health promotion to guide educational practices. J Hum Growth Dev. 2016 apr;26(1):11-20. DOI: 10.7322/jhgd.113709

20. Ruiz-Fuentes JL, Rumbaut Castillo R, Hurtado Gascón LdlC, Pastrana F. Leprosy in children: a Cuban experience on leprosy control. BMJ Paediatr Open. 2019 nov;3(1):e000500. DOI: 10.1136/bmjpo-2019-000500

21. Laurant $M$, van der Biezen $M$, Wijers $N$, Watananirun K, Kontopantelis E, van Vught AJ. Nurses as substitutes for doctors in primary care. Cochrane Database Syst Rev. 2018 jul 16;7(7):CD001271. DOI: 10.1002/14651858

22. Barreto JG, Bisanzio D, Frade MAC, Moraes TMP, Gobbo AR, Guimarães LS, et al. Spatial epidemiology and serologic cohorts increase the early detection of leprosy. BMC Infect Dis. 2015 nov;15:527. DOI:10.1186/s12879-015-1254-8

23. Goulart IMB, Santos MS, Muniz DLO, Barbosa FM, Cardoso Netto J, Chaves JGM, et al. 
Caracterização da endemia hansênica no município de Uberlândia - Minas Gerais, Brasil 1996-2000. Hansen Int [Internet]. 2006 [cited 2020 Jan 15];31(1):33-40. Available from: http:// periodicos.ses.sp.bvs.br/pdf/hi/v31n1/v31n1a05. pdf

24. Frade MAC, Paula NA, Gomes CM, Vernal S, Bernardes Filho F, Lugão HB, et al. Unexpectedly high leprosy seroprevalence detected using a random surveillance strategy in midwestern Brazil: A comparison of ELISA and a rapid diagnostic test. PLoS Negl Trop Dis. 2017 feb;11(2):e0005375. DOI: 10.1371/journal.pntd. 0005375
25. Ignotti E, Paula RC. Situação epidemiológica da hanseníase no Brasil: análise de indicadores selecionados no período de 2001 a 2010. In: Brasil. Ministério da Saúde. Secretaria de Vigilância em Saúde. Departamento de Análise de Situação em Saúde. Saúde Brasil 2010: uma análise da situação de saúde e de evidências selecionadas de impacto de ações de vigilância em saúde [Internet]. Brasília; 2011. p. 185-202 [cited 2020 Jan 15]. Available from: http://bvsms.saude.gov.br/bvs/publicacoes/ saude_brasil_2010.pdf

Recebido: 25 de setembro de 2020

Aprovado: 1 de fevereiro de 2021

Publicado: 26 de fevereiro de 2021

A Revista Baiana de Enfermagem utiliza a Licença Creative Commons - Atribuição-NãoComercial 4.0 Internacional.

https://creativecommons.org/licenses/by-nc/4.0/

Este artigo é de acesso aberto distribuído sob os termos da Licença Creative Commons (CC BY-NC).

Esta licença permite que outros remixem, adaptem e criem a partir do seu trabalho para fins não comerciais. Embora os novos trabalhos tenham de lhe atribuir o devido crédito e não possam ser usados para fins comerciais, os usuários não têm de licenciar esses trabalhos derivados sob os mesmos termos. 